\title{
Desde el ISET al InDRE. I. Instituto de Salubridad y Enfermedades Tropicales: génesis y primeros años, 1934-1940
}

Jorge Alejandro Ramírez-Hernández, ${ }^{1}$ María del Carmen Guzmán-Bracho² y José Alberto Díaz-Quiñonez ${ }^{2}$ ${ }^{1}$ Universidad Nacional Autónoma de México, Facultad de Medicina; ${ }^{2}$ Instituto de Diagnóstico y Referencia Epidemiológicos. Ciudad de México, México

\section{Resumen}

La visión del gobierno cardenista y el proceso para crear el Instituto de Salubridad y Enfermedades Tropicales, considerado estratégico para la salud pública, fueron congruentes con las acciones llevadas a cabo: el Departamento de Salubridad Pública encargó al científico más relevante de la época, Eliseo Ramírez, el diseño y seguimiento del Instituto, que una comisión del Consejo Nacional de Educación Superior y la Investigación Científica revisó, aprobó y desde su inicio le confirió el carácter de investigación con vocación social y humanística. El Instituto de Salubridad y Enfermedades Tropicales se convirtió en el primer organismo posrevolucionario que nació con plazas bien remuneradas para realizar investigación de tiempo completo en México, ofreciendo servicio clínico y enseñanza y apoyando las campañas sanitarias en la prevención y control de las enfermedades. Sin duda fue un parteaguas en la salud pública mexicana y cristalizó las expectativas de varias generaciones de médicos e investigadores en salud para tener una institución de alto nivel. Es la más significativa institución de salud pública del país, con una nueva etapa como Instituto de Diagnóstico y Referencia Epidemiológicos; sin ella no se entiende la moderna salud pública de México.

PALABRAS CLAVE: Instituto de Salubridad y Enfermedades Tropicales. Instituto de Diagnóstico y Referencia Epidemiológico. Historia de la medicina. Salud pública.

\begin{abstract}
Lázaro Cárdenas government vision, and the process to create the Sanitary and Tropical Diseases Institute, which is considered strategic for public health, were consistent with the actions that were carried out: the Department of Public Sanitary asked the most relevant scientist of those days, Eliseo Ramírez, to design and follow-up the Institute's project. A commission of the Higher Education and Scientific Research Council reviewed and approved the project and conferred this institution its nature of research center with social and humanistic vocation since its foundation. Sanitary and Tropical Diseases Institute became the first post-revolutionary health institution that was born with well-remunerated job positions to conduct full-time research in Mexico, offering clinical services, teaching and supporting disease prevention and control campaigns. It was with no doubt a milestone in Mexican public health and crystalized the expectations of several generations of physicians and health researchers about having a high-level institution. It is the most significant public health institution of the country, with a new phase as Institute of Epidemiological Diagnosis and Reference. Without it, Mexican modern public health cannot be understood.
\end{abstract}

KEY WORDS: Institute of Public Health and Tropical Diseases. Institute of Epidemiological Diagnosis and Reference. History of medicine. Public health.

Correspondencia:

María del Carmen Guzmán-Bracho

E-mail: cguzmanbracho@ hotmail.com
Fecha de recepción: 13-03-2019

Fecha de aceptación: 22-03-2019

DOI: 10.24875/GMM.19005157
Gac Med Mex. 2019;155:322-327

Disponible en PubMed

www.gacetamedicademexico.com 


\section{Introducción}

La idea inicial para la creación de un instituto de investigación de las enfermedades tropicales en México puede encontrarse en los antecedentes biográficos de Plutarco Elías Calles, quien padeció una enfermedad tropical antes de ser declarado presidente. Después de las elecciones viajó a Europa, ${ }^{1}$ a Hamburgo, para ser tratado de enfermedad de Pott o tuberculosis ósea. Concibió el proyecto de un instituto que estudiara este tipo de padecimientos. Durante su presidencia se construyó el edificio del Departamento de Salubridad Pública (1926-1928), actual Secretaría de Salud, frente a Chapultepec, pero el proyecto del instituto quedó pendiente.

Más tarde, durante la presidencia de Abelardo Rodríguez, se recibió una propuesta para la formación de un centro de investigación en salubridad pública. Los acontecimientos políticos impidieron su realización, pero Gastón Melo, jefe del Departamento de Salubridad Pública, lo dejó asentado en el capítulo correspondiente del plan sexenal..$^{2,3}$

El Partido Nacional Revolucionario, fundado en 1929 por Calles, desarrolló un plan de gobierno en 1933 y el plan sexenal de Lázaro Cárdenas se basó en esas iniciativas. En el documento oficial de ese plan, de diciembre de 1933, se anotó "como México es un país invadido por diversas enfermedades tropicales, se establecerá un instituto para el estudio de esos padecimientos". ${ }^{4}$

Otra propuesta que reforzó la anterior fue la del Primer Congreso Nacional de Higiene Rural, efectuado en Michoacán, al que asistieron Miguel E. Bustamante y Manuel Martínez Báez. Una de las recomendaciones demandaba al Departamento de Salubridad Pública que apresurara la instalación del Instituto de Salubridad y Enfermedades Tropicales. ${ }^{5}$

Al momento de la redacción del Plan Sexenal 19341940, México disponía de un Departamento de Salubridad a cargo de campañas contra padecimientos específicos: viruela, paludismo, rabia, oncocercosis, tuberculosis, enfermedades de transmisión sexual y desnutrición. ${ }^{6,7}$

A inicios de la década de 1930, la población mexicana era menor a los 17 millones de habitantes, $76 \%$ residente de área rural y más de $60 \%$ menor de 30 años; la esperanza de vida al nacimiento alcanzaba solo 33.9 años, 33.0 para hombres y 34.7 en mujeres. La mortalidad general era de 8.6 por 100000 habitantes y las tasas de mortalidad preescolar e infantil
Tabla 1. Distribución porcentual de las principales causas de muerte en los años 1931 y $1940^{\circ}$

\begin{tabular}{|ll|c|c|}
\hline \multicolumn{1}{|c|}{ Capítulo } & 1931 & 1940 \\
\hline I & Enfermedades infecciosas y parasitarias & 45.5 & 43.1 \\
\hline VIII & Enfermedades del aparato respiratorio & 17.4 & 20 \\
\hline XVI & Signos y síntomas mal definidos & 11.6 & 7.4 \\
\hline IX & Enfermedades del aparato digestivo & 4.4 & 4.6 \\
\hline XVII & Traumatismos y envenenamientos & 4.3 & 5.1 \\
\hline & Total & 83.2 & 80.2 \\
\hline
\end{tabular}

eran de 36.0 y $131.6 .^{8}$ De acuerdo con los grupos de enfermedades utilizadas en la época, más de $40 \%$ de las muertes en todo el territorio nacional en el periodo de 1931 a 1940 correspondieron a enfermedades infecciosas y parasitarias (Tabla 1).

En un desagregado de esta información se observaba que la neumonía, la diarrea y las anomalías congénitas fueron las responsables de al menos $70 \%$ de las muertes. ${ }^{9}$ Las seis primeras causas de muerte reportadas para ese año eran enfermedades infecciosas: diarrea y enteritis, neumonía e influenza, fiebre y caquexia palúdica, tos ferina, viruela y sarampión. En general, entre las primeras 20 causas de muerte, $14(70 \%)$ eran de tipo infeccioso. ${ }^{10}$ En este contexto de salud de la población mexicana nació el Instituto de Salubridad y Enfermedades Tropicales (ISET).

El propósito de este trabajo es describir y analizar el surgimiento y los primeros años, de 1934 a 1940, del Instituto a través de la consulta de fuentes primarias: se visitaron 12 archivos históricos, bibliohemerotecas y fondos fotográficos ubicados en la Ciudad de México y uno en Ginebra, Suiza. Finalmente, se analizaron los procesos fundamentales que le dieron origen.

\section{Génesis e inicio de actividades}

Con base en lo establecido en el Plan Sexenal y también referido en el Código Sanitario de los Estados Unidos Mexicanos de 1934:11

Art. 464. El Departamento de Salubridad fundará también Institutos de Higiene, para la investigación de sus problemas propios, y en especial de los padecimientos tropicales.

Para dar cauce a estas y otras iniciativas de investigación y enseñanza, el gobierno de Lázaro Cárdenas, el 25 de octubre de 1935 decretó el inicio del Consejo Nacional de Educación Superior e Investigación Científica (CNESIC). ${ }^{12}$ Su principal misión fue 
crear, transformar o suprimir establecimientos de educación superior, así como dar origen y organizar institutos que tuvieran por objeto practicar investigaciones científicas. ${ }^{13}$ De esta forma se quiso asegurar su alto nivel técnico y científico.

En la editorial del primer número de la Revista del Instituto de Salubridad y Enfermedades Tropicales ${ }^{14}$ se mencionó el trabajo de una comisión para formular el proyecto del Instituto, integrada en 1935 por Miguel E. Bustamante, Gerardo Varela, Eliseo Ramírez y Manuel Martínez Báez.

Poco después, el Departamento de Salubridad Pública, a cargo de José Siurob, le pidió a Eliseo Ramírez Ulloa desarrollar el proyecto para el Instituto de Salubridad y Enfermedades Tropicales, además de nombrarlo representante del Departamento de Salubridad Pública ante ese Consejo. ${ }^{15}$ Eliseo Ramírez Ulloa era el más destacado científico médico de la época ${ }^{16}$ y jefe del Laboratorio Central (surgido en 1929).

Como resultado de este trabajo, el 24 de marzo de 1936 se hizo entrega oficial al CNESIC del "Proyecto de Organización del Instituto de Salubridad y Enfermedades Tropicales"17 y Ramírez lo informó al pleno del Consejo durante la sesión del 16 de abril.18

La revisión del proyecto quedó a cargo de una comisión del CNESIC integrada por Rafael Illescas Frisbie, Juan O'Gorman, Alonso M. Jaimes y Eliseo Ramírez Ulloa. ${ }^{19,20}$ Después de dos meses de trabajo, el proyecto fue aprobado el 27 de junio de 1936, de acuerdo con la minuta de la sesión del CNESIC. ${ }^{21}$ Eliseo Ramírez, como representante del Departamento de Salubridad Pública ante el Consejo, dio respuesta a las observaciones del proyecto:

El Departamento de Salubridad, creo yo, no tendrá inconveniente, pues no variándose lo fundamental del proyecto ni de los programas, y siendo esto solo una cuestión de redacción estará conforme.

El proyecto fue aprobado por el pleno a las 14:00 horas y la minuta fue firmada por Enrique Díaz de León y Enrique Arreguín, presidente y secretario del CNESIC, respectivamente. ${ }^{22}$ Con esta decisión se continuaron los trabajos del ISET plasmados en el Plan Sexenal. Con el respaldo del dictamen emitido por el Consejo, el ISET se convirtió en la primera institución en el México posrevolucionario que nació completa con plazas asignadas para la investigación en salud, espacio clínico con hospitalización para dar servicio a la población, además de apoyar a las campañas sanitarias e impartir docencia en la Escuela de
Salubridad. Todo en un ambiente institucional con valores humanísticos: ${ }^{23}$

Teniendo en cuenta que es condición indispensable, para que el trabajo científico sea fecundo, la continuidad del esfuerzo, el Consejo estima de importancia fundamental que debe garantizarse a los investigadores la justa inamovilidad y una decorosa retribución,... y que necesita, además, tranquilidad para dedicarse por entero, sin cortapisas, al estudio de los asuntos que el Estado confía a su inteligencia y esfuerzo.

Una semana después de la sesión se informó vía oficial a José Siurob, la aprobación definitiva del Proyecto de Organización y Programas Generales del Instituto de Salubridad y Enfermedades Tropicales, con referencia a las modificaciones introducidas. ${ }^{24}$

\section{Inicio de actividades}

Para entender el inicio de actividades del ISET se sugiere que el Instituto estaba preparado para su inauguración formal en 1938, de hecho, los periódicos lo anunciaban desde 1937.

La trascendencia del proyecto aprobado motivó la difusión de la noticia a la población, de acuerdo con el oficio enviado por el CNESIC a los periódicos de mayor circulación en la época. Así, El Universal dio a conocer el dictamen el día 7 de julio de 1936:25

Al fin se fundará...

El Instituto de Salubridad y Enfermedades Tropicales se ocupará del estudio de los problemas técnicos de la salubridad relacionados con las enfermedades tropicales existentes en la República Mexicana y en las zonas colindantes, y de aquellos que por su índole puedan ser abordados en un instituto de esta naturaleza.

Para llenar su objeto, el Instituto procederá a desarrollar y a sistematizar los trabajos que en la actualidad se efectúan sin coordinación en diferentes dependencias del Departamento. Emprenderá trabajos de investigación acerca de temas actualmente en estudio y de otros nuevos, principalmente de los referentes a la investigación y al estudio de padecimientos de existencia probable o posible en nuestra zona tropical. Complementariamente se dedicará a la investigación acerca de la higiene de la alimentación en los centros rurales; estudiará las intoxicaciones producidas por animales ponzoñosos y por plantas, y servirá de institución docente y centro de consulta.

Para realizar sus funciones, contará con laboratorios de investigación, sección clínica de hospitalización, biblioteca, museo, granja para animales de laboratorio y las dependencias administrativas necesarias.

En su local alojará a la Escuela de Salubridad del Departamento, a la cual dará la ayuda necesaria para el mayor desempeño de su cometido. La Escuela de Salubridad tendrá el personal que el Departamento señala y las relaciones de esta institución con el Instituto se fijarán por la reglamentación especial que formulará el Departamento. 
Otro argumento del comienzo de actividades previo a la inauguración formal lo constituye una fotografía en donde se ve la disposición de las letras metálicas en la pared principal con la fecha "Septiembre de MCMXXXVIII" (Figura 1). ${ }^{26}$

Con un edificio terminado en su construcción ${ }^{27}$ y un proyecto de organización y funcionamiento autorizado de acuerdo con las disposiciones presidenciales, fue necesario volcar esfuerzos para la compra de mobiliario administrativo y de laboratorio, así como del equipo e insumos para las áreas técnicas y empezar a contratar investigadores. Para esta tarea, Suirob designó de nuevo a Eliseo Ramírez. Se proporcionaron al ISET equipos e instrumentos de gran calidad y de vanguardia. La mayoría de estos recursos fueron obtenidos a través de trueque de petróleo con Alemania, ${ }^{28}$ insólito resultado que la nacionalización dejó a México al limitarse el acceso a mercados internacionales para la venta del crudo, no así con el país teutón ávido de petróleo para el conflicto que preparaba. Para 1982, aún se encontraban en el ISET infinidad de etiquetas alemanas en muchos de los equipos y reactivos utilizados todavía en los laboratorios (referencia personal de María del Carmen Guzmán Bracho).

Sin embargo, la nacionalización petrolera, precipitada por los eventos en Europa (la toma de Austria por Hitler), ${ }^{29}$ se convirtió en la prioridad presidencial, lo cual llevó a postergar la apertura formal del ISET hasta 1939.

\section{Actividades de Eliseo Ramírez}

El Instituto inició actividades a principios de 1938, mientras se llevaba el proceso de invitación a investigadores, recepción de equipo e insumos de laboratorio y contratación de personal, como lo narra uno de los investigadores en sus memorias, Enrique Beltrán. ${ }^{28}$ La Revista de la Oficina Sanitaria Panamericana de ese año, ${ }^{30}$ con base en información del Departamento de Salubridad Pública, dio a conocer el acontecimiento y el nombre de su director:

...que se ocupará del estudio de los problemas técnicos relacionados con las enfermedades tropicales existentes en la República Mexicana y en las zonas colindantes, y de las otras que por su índole pueden ser abordadas en un establecimiento de esa naturaleza. El Instituto comenzará por desarrollar y sistematizar los trabajos que en la actualidad se efectúan en diferentes dependencias del Departamento de Salubridad. Emprenderá igualmente trabajos de investigación pertinente, incluso higiene de los alimentos en los centros rurales, intoxicaciones producidas por

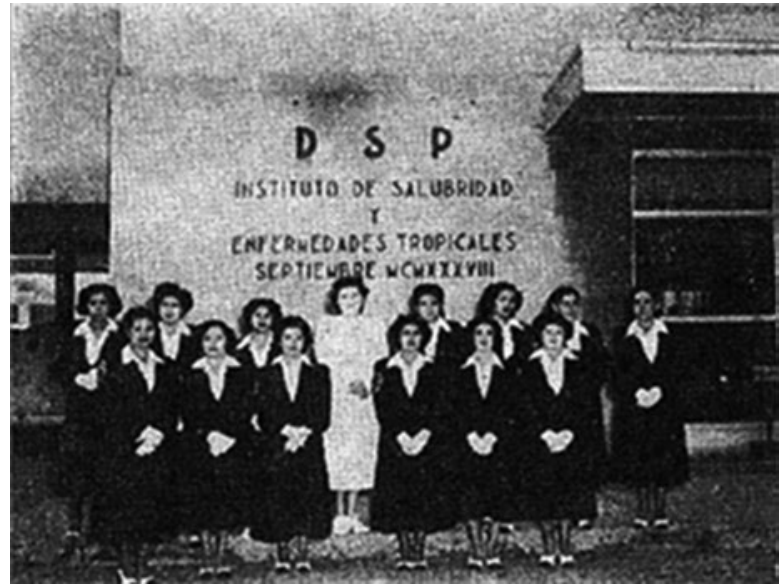

Figura 1. Inscripción en letras metálicas en la fachada principal del edificio del Instituto de Salubridad y Enfermedades Tropicales, donde se reconoce el nombre del Instituto, su adscripción al Departamento de Salubridad Pública, con la fecha septiembre de 1938.

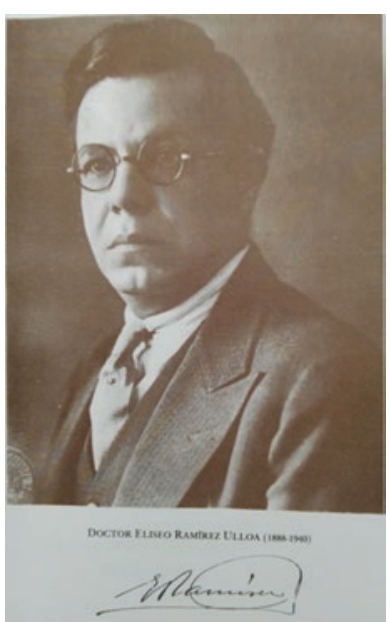

Figura 2. Eliseo Ramírez Ulloa. ${ }^{16}$

animales ponzoñosos y por plantas, y servirá igualmente de centro docente y de centro de consultas... director ha sido nombrado Eliseo Ramírez (Inf. Dpto. Sal. Púb.).

El presidente Lázaro Cárdenas autorizó la creación de plazas especiales para los jefes de laboratorio del ISET con la denominación de investigador científico A, con una asignación de $\$ 900.00$ mensuales, ${ }^{31}$ que constituían una elevada remuneración en la época.

Eliseo Ramírez, como se ha mencionado, participó desde el inicio en diseñar el proyecto del ISET y someterlo ante el CNESIC, donde además fue representante del Departamento de Salubridad Pública. Después de la aprobación comenzó a equipar y a contratar al personal de investigación del Instituto. En ese periodo, presumiblemente por enfermedad, dejó estas funciones y regresó en agosto de 1940 como director. Falleció en funciones el 28 de diciembre de 1940 (Figura 2). 
Tabla 2. Formación académica de los investigadores fundadores del Instituto de Salubridad y Enfermedades Tropicales

\begin{tabular}{|c|c|c|}
\hline Investigador & Área & Formación académica \\
\hline Alberto P. León & Bacteriología e Inmunología & Médico cirujano (UNAM), maestría en Salud Pública (Harvard) \\
\hline Antonio González Ochoa & Micología & $\begin{array}{l}\text { Médico cirujano (UNAM), médico colonial (París), diplomado en Micología } \\
\text { Médica (París), curso especializado (Alemania) }\end{array}$ \\
\hline Efrén C. Del Pozo Rangel & Terapéutica experimental & Médico cirujano (UNAM) \\
\hline Eliseo Ramírez Ulloa & Fisiología y farmacología & Médico cirujano (UNAM), doctor en Ciencias (UNAM) \\
\hline Enrique Beltrán Castillo & Protozoología & Profesor en Ciencia Naturales (UNAM), PhD (Columbia, Estados Unidos) \\
\hline Esther Luque Muñoz & Botánica & Profesor en Farmacia (UNAM) \\
\hline Gerardo Varela Mariscal & Bacteriología & $\begin{array}{l}\text { Médico cirujano (UNAM), posgrado en Microbiología (Instituto Pasteur, H. Cochin } \\
\text { París), curso en Salud Pública (Harvard) }\end{array}$ \\
\hline Luis Mazzotti Galindo & Helmintología & $\begin{array}{l}\text { Médico cirujano (UNAM), curso en Salud Pública (John Hopkins), diplomado en } \\
\text { Medicina Tropical (Liverpool) }\end{array}$ \\
\hline Luis Vargas García Alonso & Entomología & $\begin{array}{l}\text { Médico cirujano (UNAM), maestro en Ciencias Biológicas (UNAM), curso en } \\
\text { Salud Pública (John Hopkins) }\end{array}$ \\
\hline Manuel Martínez Báez & Anatomía patológica & $\begin{array}{l}\text { Médico cirujano (Michoacán), médico malariólogo (París) y cursos } \\
\text { especializados en Italia, España y Alemania }\end{array}$ \\
\hline Miguel E. Bustamante V. & Epidemiología & Médico cirujano (UNAM), PhD (John Hopkins)) \\
\hline Teófilo García Sancho & Química & Ingeniero químico (UNAM) y PhD (Kiel) \\
\hline
\end{tabular}

\section{La producción científica ${ }^{32}$}

Los investigadores fundadores fueron identificados para su incorporación al Instituto por su sólida formación académica y amplia experiencia en el área de interés (Tabla 2).

Desde sus inicios, la producción de la investigación se manifestó en la vasta publicación de artículos por parte de los investigadores de ISET. Más aún, a partir del segundo año de labores, el Instituto contó con una revista propia editada por la Dirección de Educación Higiénica del Departamento de Salubridad Pública que...

...publica los trabajos de investigación realizados por el personal del establecimiento y acepta colaboraciones de especialistas nacionales y extranjeros.

El consejo de redacción lo integraron inicialmente Manuel Martínez Báez y Enrique Beltrán Castillo. El primer número de la Revista del Instituto de Salubridad y Enfermedades Tropicales, que apareció con fecha del último trimestre de 1939, publicó trabajos iniciales realizados por los investigadores del ISET. Esta revista hizo acopio de los productos de investigación de los distintos grupos de trabajo del Instituto en 22 artículos, en los que es posible identificar el enfoque institucional dirigido hacia los principales problemas de salud en el país: 10 artículos sobre enfermedades parasitarias (ocho sobre paludismo y protozoarios relacionados y dos sobre enfermedad de Chagas), cinco artículos centrados en el tema entomológico (anofelinos y otros mosquitos) y tres reportes de datos de interés epidemiológico como distribuciones de población, tablas de vida y causas de muerte, lo cual indica la relevancia de esta revista para el Departamento de Salubridad en ese momento. En el periodo 1939-1946, los investigadores del Instituto publicaron 224 artículos en la Revista. Se identifica el liderazgo académico de la institución dirigida a investigar y resolver los principales problemas de salud: Luis Vargas publicó 37 artículos, Luis Mazzotti 31, Beltrán 30, Bustamante 23, Varela 26, Zozaya 25, León 19 y González Ochoa 13, quien se incorporó en 1940 al Instituto.

\section{Comentarios finales}

En este artículo se han mencionado, respecto al proyecto del Instituto, dos comisiones. La primera de 1935, citada antes en el artículo anónimo en la editorial del primer número de la revista del ISET. ${ }^{14}$ En nuestro trabajo de investigación no se ha encontrado ninguna referencia primaria al respecto.

Por otro lado, se ha documentado el encargo del Departamento de Salubridad Pública a Eliseo Ramírez como su representante en el CNESIC ${ }^{15}$ (quien rechazó la remuneración adicional ofrecida por este 
Consejo ${ }^{33}$ ), su responsabilidad para obtener la aprobación del proyecto y los primeros pasos de organización del ISET.

El otro punto para aclarar es el año de inicio de actividades. Hay amplia evidencia que desde 1938 el Departamento de Salubridad Pública informaba a través del Boletín de la Oficina Sanitaria Panamericana el inicio de los trabajos del ISET y daba el nombre del director, Eliseo Ramírez, quien había recibido el encargo de diseñar y asegurar la aprobación del CNESIC y posteriormente la función de organizar, equipar y contratar investigadores, como lo escribe con precisión el testimonio de Enrique Beltrán.

Además del carácter científico, es importante hacer énfasis en el carácter humanístico del ISET. Como ejemplos encontramos los trabajos de Miguel E. Bustamante en la historia de la salud pública, los ensayos filosóficos de Eliseo Ramírez y sobre los aspectos culturales de las enfermedades tropicales de Martínez Báez.

Sin duda, el ISET fue un parteaguas en la salud pública del país, ${ }^{34}$ cristalizó las expectativas de varias generaciones de médicos e investigadores en salud, para tener una institución de alto nivel. En su nueva etapa, de Instituto de Diagnóstico y Referencia Epidemiológicos (InDRE), es la más significativa institución de salud pública del país, sin ella no se entiende la moderna salud pública de México.

\section{Agradecimientos}

A las autoridades y personal del Archivo Histórico de la Secretaría de Salud. Al doctor en Historia Andrés Ortiz Morales, subdirector del Archivo Histórico del Instituto Politécnico Nacional.

\section{Bibliografía}

1. Valenzuela GJ. El viaje de Plutarco Elías Calles como presidente electo por Europa y Estados Unidos. Rev Mex Sociol.1995;57:191-210.
2. Fondo CNESIC. C1E20F23. México: Archivo General de la Nación; 2018.

3. Melo G. Tercer director de la Escuela de Salubridad, 1926-1928. Bol Salubridad Mex. 1933;4:1-2.

4. Cárdenas-Del Río L. PNR: plan sexenal 1934-1940. México: Partido Nacional Revolucionario; 1933.

5. Oikión-Solano V. Primer Congreso Nacional de Higiene Rural celebrado en Morelia, del 3 al 12 de noviembre de 1935. Relaciones (COLMICH). 1993;14:273-303.

6. Rodríguez ME. Aspectos médicos-sanitaristas en México (1934-1940). Gac Med Mex. 2017;153:609-612.

7. Viesca-Treviño C. Campañas contra enfermedades durante el cardenismo. Gac Med Mex. 2017;153:613-616.

8. Kumate J. La salud de los mexicanos. 1929-2000. México: El Colegio Nacional; 2010.

9. Estadísticas históricas de México. Tomo I. México: Instituto Nacional de Estadística, Geografía e Informática; 1994.

10. Compendio histórico. Estadísticas vitales 1893-1993. México: Secretaría de Salud; 2004

11. Código Sanitario de los Estados Unidos Mexicanos. Diario Oficial de la Federación 1934 Ago 31. p. 1164-1200.

12. Decreto que crea el Consejo Nacional de la Educación Superior y la Investigación Científica. México: Diario Oficial de la Federación; 1935 Oct 30. p. 1078-1079.

13. Archivo General de la Nación. Fondo CNESIC. C1E9F4. México: Archivo General de la Nación; 2018.

14. Anónimo. El Instituto de Salubridad y Enfermedades Tropicales. Revista del Instituto de Salubridad y Enfermedades Tropicales. 1939;1:3-35.

15. Fondo CNESIC. C21E16. México: Archivo General de la Nación; 2018.

16. Kumate J. Semblanza del Dr. Eliseo Ramírez Ulloa. En: Eliseo Ramírez Ulloa. Obras Completas. Tomo II. Investigación Médica. México: El Colegio Nacional; 1988.

17. Fondo CNESIC. C2E16F3. México: Archivo General de la Nación; 2018.

18. Fondo CNESIC. C1E20F14. México: Archivo General de la Nación; 2018.

19. Fondo CNESIC. C1E20F30. México: Archivo General de la Nación; 2018.

20. Fondo CNESIC. C1E20F33. México: Archivo General de la Nación; 2018.

21. Fondo CNESIC. C1E20F59. México: Archivo General de la Nación; 2018.

22. Fondo CNESIC. C1E20F66. México: Archivo General de la Nación; 2018.

23. Fondo CNESIC. C2E16F86. México: Archivo General de la Nación; 2018.

24. Fondo CNESIC. C2E16F76. México: Archivo General de la Nación; 2018.

25. Instituto de Salubridad y Enfermedades Tropicales. Al fin se fundará. El Universal 1936 Jul 7. Primera plana.

26. Archivo Histórico de la Secretaría de Salud. Anuario de la Escuela de Salubridad e Higiene. México: Secretaría de Salubridad y Asistencia; 1952.

27. Andreu-Almazán L. La sanidad en México. Bol Oficina Sanitaria Panamericana. 1939;18:617-621.

28. Beltrán E. Medio siglo de recuerdos de un biólogo mexicano. México: Sociedad Mexicana de Historia Natural; 1977.

29. Cárdenas-Del Río L. Obras: Apuntes 1913/1940. Tomo I. México: Universidad Nacional Autónoma de México; 1972.

30. Boletín de la Oficina Sanitaria Panamericana. México: Oficina Sanitaria Panamericana/Instituto Mexicano de Investigación; 1938.

31. Fondo CNESIC. C20E5. México: Archivo General de la Nación; 2018.

32. Colección de la Revista del Instituto de Salubridad y Enfermedades Tropicales del Instituto de Diagnóstico y Referencia Epidemiológicos. México: Instituto de Diagnóstico y Referencia Epidemiológicos.

33. Fondo CNESIC. C7E16. México: Archivo General de la Nación; 2018.

34. Santos-Preciado JI, Franco-Paredes C. Enfermedades tropicales del rezago: a 72 años del establecimiento del Instituto de Salubridad y Enfermedades Tropicales en México. Bol Med Hosp Infant Mex. 2011; 68:83-85 